\title{
Temporal extension of stable glow discharges in fluorine-based excimer laser gas mixtures by the addition of xenon
}

\author{
D. Mathew · P.J.M. Peters • H.M.J. Bastiaens • \\ K.-J. Boller
}

Received: 31 January 2008 / Revised version: 11 February 2009 / Published online: 4 March 2009

(C) The Author(s) 2009. This article is published with open access at Springerlink.com

\begin{abstract}
The effect of addition of xenon on the long term homogeneity of discharges in $\mathrm{F}_{2}$ and $\mathrm{ArF}$ excimer laser gas mixtures was investigated in a small-volume discharge chamber. The gas mixture in the discharge chamber was preionized by X-rays. A special electrical excitation circuit containing a pulse forming line provided a long, squareshaped current pumping pulse of a predetermined duration to the discharge electrodes. The initiation and the development of the discharge was monitored via its fluorescence signal with an intensified CCD camera. We found that adding Xe up to partial pressures of 0.53 mbar extended the homogeneous phase of the discharge from $80 \mathrm{~ns}$ to approximately $200 \mathrm{~ns}$ in $\mathrm{He} / \mathrm{F}_{2}$ as well as in $\mathrm{He} / \mathrm{Ar} / \mathrm{F}_{2}$ and $\mathrm{Ne} / \mathrm{Ar} / \mathrm{F}_{2}$ excimer laser gas mixtures. Monitoring of the $\mathrm{ArF}$ and $\mathrm{XeF}$ spontaneous emission signals showed that the formation of ArF excimers remained unaffected by the addition of xenon (up to 1.3 mbar) to the laser gas mixture.
\end{abstract}

PACS 42.55.Lt $\cdot$ 42.60.Lh $\cdot 52.80 .-\mathrm{s}$

\section{Introduction}

Fluorine-based excimer lasers such as $\mathrm{KrF}, \mathrm{ArF}$ and $\mathrm{F}_{2}$ are generally pumped by pulsed high-pressure transversal glow discharges. However, such discharges tend to become instable with time, which manifests itself in filamentation, i.e., the formation of thin channels of enhanced current density within the discharge. Eventually, a homogeneous discharge

D. Mathew · P.J.M. Peters $(\bowtie) \cdot$ H.M.J. Bastiaens $\cdot$ K.-J. Boller Laser Physics and Nonlinear Optics Group, MESA + Research Institute, Faculty of Science and Technology, University of Twente, P.O. Box 217, 7500 AE Enschede, The Netherlands e-mail: P.J.M.Peters@tnw.utwente.nl will turn into an arc that bridges the electrode gap [1,2]. Such filamentation or arcing in a discharge deteriorates the optical homogeneity of the gain medium [3,4], resulting in a termination of the laser pulse before the end of the current pump pulse, thereby limiting the maximum laser pulse duration to 20-30 ns. As a consequence, the laser beam contains mainly radiation of low spatial coherence originating from amplified spontaneous emission, whereas in many industrial applications of excimer lasers it is desired to have a diffraction-limited, high-quality laser beam. For instance, in material processing, a high beam quality is required for high resolution structuring and drilling. A high beam quality can be obtained only when a homogeneous gain medium is present for several hundreds of nanoseconds, allowing the laser beam a sufficient number of round trips in the resonator in order to suppress unwanted higher-order modes in the output. Maintaining a stable, homogeneous discharge for up to hundreds of nanoseconds is therefore an important issue, requiring the elimination or suppression of discharge instabilities. Kataoka et al. [5] have shown that the addition of small amounts of various gases, in particular xenon (at ppm level) in ArF lasers excited with short current pulses ( $<30 \mathrm{~ns}$ ), improved the output energy by a factor of up to three, while addition of larger amounts of Xe decreased the output energy. Besaucele et al. [6] claimed in their patent that by adding very small quantities of oxygen, xenon or radon to $\mathrm{ArF}$ or $\mathrm{KrF}$ laser gas mixtures an enhanced dose control and reproducibility could be obtained for laser pulse lengths of 15-30 ns. H owever, it appeared that the total output energy was always slightly lower when xenon was added. Tanaka et al. [7] claimed that they improved a long pulse (not less than $40 \mathrm{~ns}$ ) gas laser apparatus for lithography over the prior art in the effects of the addition of xenon gas by increasing the output energy in the second half of each laser pulse when the xenon containing laser gas mixture was 
heated. In this way they produced laser pulse widths up to 60 ns. Recently Terashima et al. [8] claimed in their patent (issued on a continued prosecution application filed originally in 1999 and also based on systems with pulse durations of approximately $30 \mathrm{~ns}$ ) that the burst and spike characteristics in an excimer laser are efficiently improved by adding small amounts of Xe to a halogen containing laser gas mixture. In none of these publications a word is mentioned on discharge improvements on the longer time scales, due to the addition of xenon as for example having pulse durations above $100 \mathrm{~ns}$.

In this paper we present the results of our experiments on high-pressure gas discharges in fluorine-based excimer laser gas mixtures driven by, compared to all previous published work, very long excitation pulses (>200 ns). For this reason we used an electric-discharge circuit that was totally different compared to the usual excimer discharge excitation techniques. It was based on a pulse forming line technique that provided a long and square-shaped current pulse to the discharge electrodes once the discharge was switched on. In this way, we were able to produce a long lasting and uni-polar or non-oscillating discharge between the electrodes. Although we knew about the initial beneficial effects as mentioned previously [5-8] of small amounts of xenon added to excimer laser gas mixtures, we, for the first time, could show that there were also strong effects on the discharge stability later on in the pulse. We found that the time interval over which the discharge remained homogeneous increased significantly in $\mathrm{F}_{2}$ and ArF excimer laser gas mixtures by adding small amounts of xenon. It was shown that the addition of 0.08 mbar Xe (60 mTorr) to a $F_{2}$ laser gas mixture composed of 2 bar He and 1 mbar $^{F_{2}}$ stabilised the discharge for over $200 \mathrm{~ns}$ when operated at a pump power density of $500 \mathrm{~kW} \mathrm{~cm}^{-3}$. By adding $0.53 \mathrm{mbar}$ (400 mTorr) $\mathrm{Xe}$ to ArF laser gas mixtures, composed of 2 bar He (or $\mathrm{Ne}$ ), 60 mbar Ar and 1.5 mbar $_{2}$, we extended the duration of the homogeneous phase of the discharge from $80 \mathrm{~ns}$ to about $160 \mathrm{~ns}$ at a power density of $1.3 \mathrm{MW} \mathrm{cm}^{-3}$. Simultaneous monitoring of the ArF (193 nm) and XeF (351 nm) fluorescence signals from discharges in ArF laser gas mixtures showed that the addition of Xe did not perturb the formation of ArF excimer molecules. It is therefore expected that the production of stable glow discharges for such extended durations will enable the development of long pulse $F_{2}$ and ArF excimer lasers with high beam quality.

\section{Experimental setup}

The experiments have been performed using the experimental set-up described earlier in [9, 10]. Briefly, the set-up comprised a small quartz discharge chamber with two nickel coated aluminum electrodes placed $17 \mathrm{~mm}$ apart. The gas mixture was pre-ionised by a short burst $(\approx 17 \mathrm{~ns})$ of highintensity $\mathrm{x}$-rays. Discharges have been generated in typical excimer laser gas mixtures of 2 bar He/1 mbar $\mathrm{F}_{2}$ and 2 bar $\mathrm{He}$ (or $\mathrm{Ne}$ )/60 mbar Ar/1.5 mbar $\mathrm{F}_{2}$ to which a varying amount of $\mathrm{Xe}$ (0 to $1.3 \mathrm{mbar}$ ) was added. The spatiotemporal development of the discharge was monitored via the fluorescence signal from the discharge using an intensified CCD camera (ICCD camera, 4 Picos, Stanford Computer Optics). The spatial resolution of the recorded images was about $120 \mu \mathrm{m}$. The gating time of the ICCD camera was set to $2 \mathrm{~ns}$ for all measurements. Two photomultiplier tubes (Hamamatsu R331, Philips 56AVP) equipped with the respective band pass filters have been used to detect the spontaneous emission signals from the ArF (193 nm) and XeF (351 nm) excimers formed during the discharge.

\section{Experimental results}

The effect of the addition of Xe on the homogeneity of the discharge in a $\mathrm{F}_{2}$ excimer laser gas mixture of 2 bar $\mathrm{He}$ and 1 mbar $F_{2}$ is shown in the images displayed in Fig. 1. These images have been recorded in consecutive shots taken at $30 \mathrm{~ns}, 120 \mathrm{~ns}$ and $170 \mathrm{~ns}$ after the initiation of the discharge. In all discharge images shown, the cathode was located on top and the anode at the bottom. The current density and the pump power density in these measurements were approximately $140 \mathrm{~A} \mathrm{~cm}^{-2}$ and $500 \mathrm{~kW} \mathrm{~cm}^{-3}$, respectively, and were kept constant using current limiting resistors in series with the discharge. Figure 1(a) shows images of the discharge in 2 bar He/1 mbar $\mathrm{F}_{2}$ gas mixtures without xenon. From the image taken $30 \mathrm{~ns}$ after discharge initiation, it can be seen that the discharge started homogeneously. However, at $120 \mathrm{~ns}$ the discharge uniformity was destroyed and filaments appear in the bulk of the discharge volume. Later, at

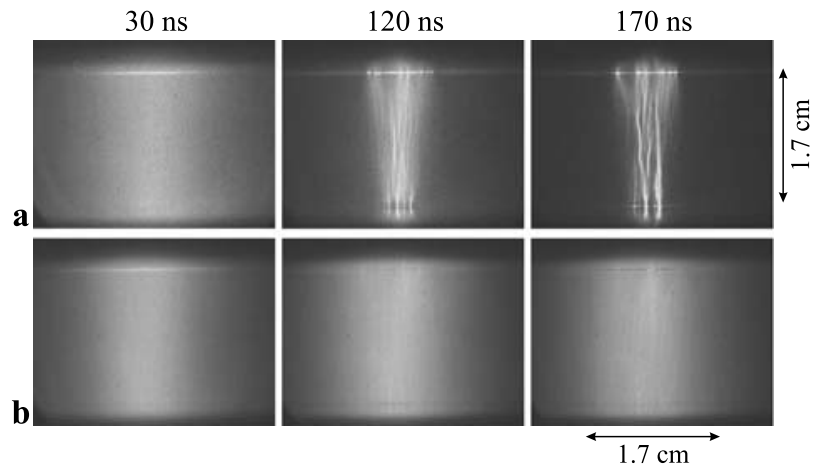

Fig. 1 Images showing the time evolution of discharges in mixtures of 2 bar $\mathrm{He}$ and 1 mbar $\mathrm{F}_{2}$, with and without xenon addition: (a) without $\mathrm{Xe},(\mathbf{b})$ with 0.08 mbar (60 mTorr) Xe added. The images have been recorded at $30 \mathrm{~ns}, 120 \mathrm{~ns}$ and $170 \mathrm{~ns}$ after the initiation of the discharge. The gating time of the ICCD camera was set to $2 \mathrm{~ns}$. The current density in these discharges was about $140 \mathrm{~A} \mathrm{~cm}^{-2}$ and the pump power density was about $500 \mathrm{~kW} \mathrm{~cm}^{-3}$. The dark areas in the upper and lower part of the images are the cathode and anode, respectively 
Fig. 2 Temporal behavior of the discharge voltage, current and power deposition in

(a) 2 bar $\mathrm{He} / 1.5$ mbar

$\mathrm{F}_{2} / 60$ mbar Ar without Xe, with 0.53 mbar Xe and with 1.3 mbar $\mathrm{Xe}$, (b) 2 bar Ne/1.5 mbar $\mathrm{F}_{2} / 60$ mbar Ar without $\mathrm{Xe}$ and with 0.53 mbar Xe. The pump power density and the current density for (a) was about $1.3 \mathrm{MW} \mathrm{cm}^{-3}$ and $340 \mathrm{~A} \mathrm{~cm}^{-2}$ and for (b), the corresponding values were about $410 \mathrm{~kW} \mathrm{~cm}^{-3}$ and $390 \mathrm{~A} \mathrm{~cm}^{-2}$

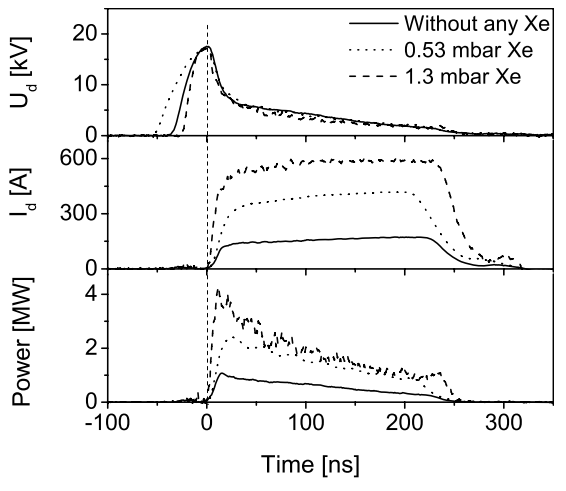

a

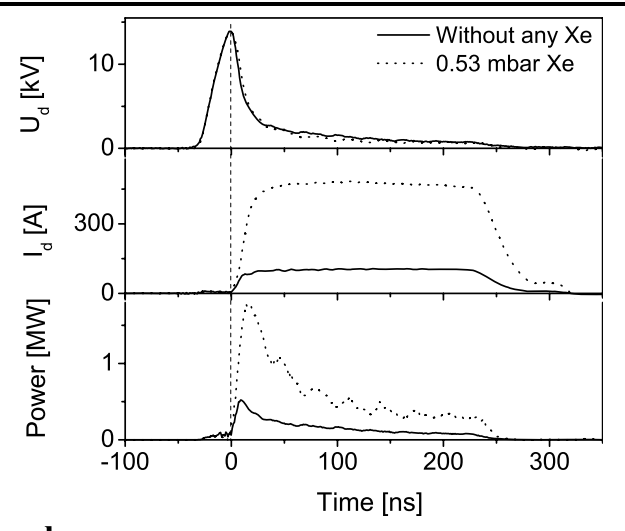

b
$170 \mathrm{~ns}$, the discharge was constricted into a few intense filaments. Figure 1(b) shows the images of the discharge after adding 0.08 mbar (60 mTorr) of Xe to the gas mixture. It is clear from these images that xenon had a very beneficial effect on the discharge homogeneity especially as the discharge developed in time. Although not shown in Fig. 1, images taken later in time showed that the discharge stayed uniform even up to the end of the current pump pulse at $220 \mathrm{~ns}$. As for $\mathrm{F}_{2}$ laser gas mixtures, we investigated the discharge homogeneity also for ArF laser gas mixtures to which Xe was added up to a partial pressure of 1.33 mbar. The measurements have been performed using a gas mixture composed of $\mathrm{He}$ or $\mathrm{Ne}$ as buffer gas, $60 \mathrm{mbar} \mathrm{Ar}$ and $1.5 \mathrm{mbar} \mathrm{F}_{2}$ at a total pressure of 2 bar. Figure 2(a) shows the temporal evolution of the discharge voltage, current and pump power for He buffered gas mixtures, without $\mathrm{Xe}$ and with addition of 0.53 mbar and 1.3 mbar Xe. It can be seen that the presence of Xe lowers the breakdown voltage slightly from $17.5 \mathrm{kV}$ to $17.2 \mathrm{kV}$. The steady state voltage $(5.9 \mathrm{kV})$, on the other hand, was not affected and remained the same during the entire pump pulse duration for both gas mixtures without and with 0.53 mbar Xe. However, this value was slightly lower $(5.7 \mathrm{kV})$ for mixtures with $1000 \mathrm{mTorr}$ Xe. During the pump pulse the steady state voltage decreased from approximately $6 \mathrm{kV}$, immediately after breakdown, to $2 \mathrm{kV}$ at the end of the pulse. From the ICCD images (shown in Fig. 3), it could be seen that the discharge width increased considerably with the amount of Xe added, which as a consequence, influenced the current density of the discharge. To keep the current density at the same value of $340 \mathrm{~A} \mathrm{~cm}^{-2}$ (at about $30 \mathrm{~ns}$ after the initiation of the discharge) in both gas mixtures with and without $\mathrm{Xe}$, the current in the gas mixture with Xe was raised from $150 \mathrm{~A}$ to about $530 \mathrm{~A}$ (by varying the resistance placed in series with the discharge). This resulted in a corresponding increase of the total power deposition. The pump power density $30 \mathrm{~ns}$ after the start of the discharge was approximately $1.3 \mathrm{MW} \mathrm{cm}^{-3}$. Figure 2(b) shows the typical waveforms of the voltage, current and power deposition for discharges in a $\mathrm{Ne}$ buffered gas mixture to which
$0.53 \mathrm{mbar} \mathrm{Xe}$ is added, and in the same gas mixture without $\mathrm{Xe}$. The breakdown voltage was found to be equal for both gas mixtures, while the steady state voltage for the discharge in the mixture with Xe was slightly lower. To keep the current density at $390 \mathrm{~A} \mathrm{~cm}^{-2}$ for the mixture with and without $\mathrm{Xe}$, the discharge current was raised from $100 \mathrm{~A}$ to $450 \mathrm{~A}$ in the same way as described above. Correspondingly, the pump power rose by more than a factor of four. The power density $\left(\sim 410 \mathrm{~kW} \mathrm{~cm}^{-3}\right)$ in $\mathrm{Ne}$ buffered gas mixtures was lower than the values of He buffered gas mixtures due to the lower steady state voltage of these discharges.

Figure 3 shows the dramatic improvement of the discharge uniformity when 0.53 mbar Xe was added to He and Ne buffered gas mixtures. The discharge images have been taken in consecutive shots at $30 \mathrm{~ns}, 80 \mathrm{~ns}$ and $160 \mathrm{~ns}$ after the initiation of the discharges. Figure 3(a) shows images of the discharges in a He buffered ArF excimer laser gas mixture without Xe. It can be seen that weak filaments have been formed as early as $30 \mathrm{~ns}$ after the initiation of the discharge and that these filaments became more intense with time. At about $80 \mathrm{~ns}$ the discharge became rather nonuniform. By $160 \mathrm{~ns}$, current filaments have taken over the homogeneous discharge glow and the homogeneity was deteriorated completely. Another feature to be noted is that the width of the discharge reduced considerably towards the end of the pump pulse. To this He buffered ArF laser gas mixture, varying amounts of $\mathrm{Xe}(0.027 \mathrm{mbar}$ to $1.3 \mathrm{mbar})$ have been added in steps. For Xe additions up to partial pressures of $0.053 \mathrm{mbar}$, no notable improvement of the discharge uniformity was observed (images not shown here). However, when the amount of $\mathrm{Xe}$ was raised further to $0.13 \mathrm{mbar}$ (100 mTorr), the discharge homogeneity increased noticeably and after a discharge duration of about 100 ns thin current filaments appeared (images not shown here). A further increase of the Xe partial pressure to $0.53 \mathrm{mbar}$, resulted in a further improvement of the discharge homogeneity which was preserved for a longer time (160 ns) as is shown in Fig. 3(b). From the image taken at $30 \mathrm{~ns}$ it can be seen that the discharge started very homogeneously, and that at ap- 


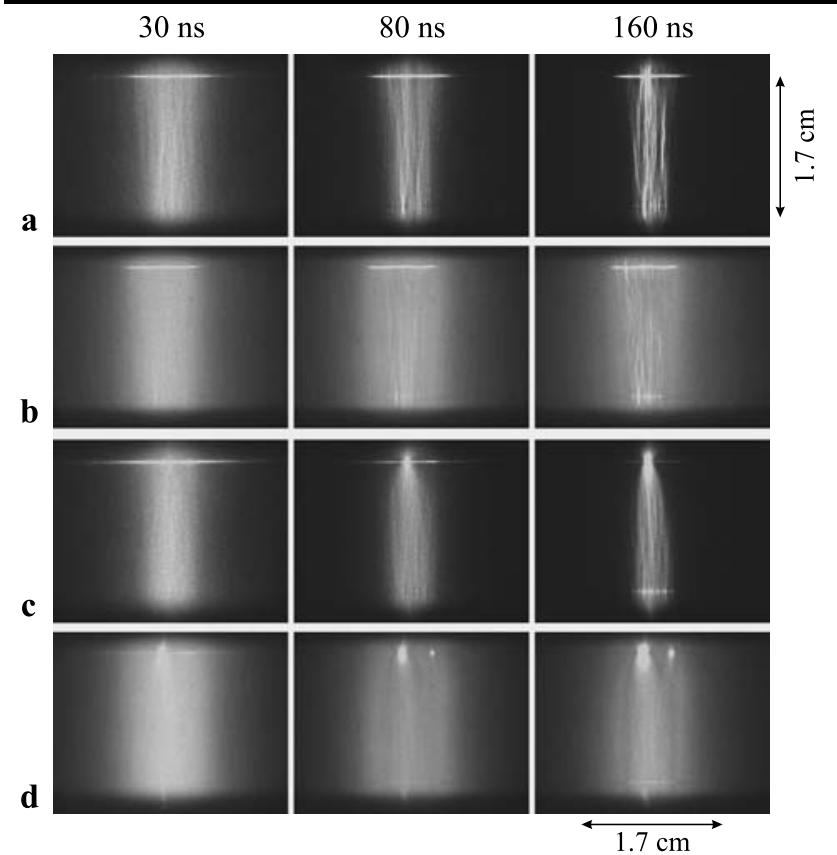

Fig. 3 Images showing the time evolution of discharges in ArF excimer laser gas mixtures, with and without xenon addition. The images are taken at $30 \mathrm{~ns}, 120 \mathrm{~ns}$ and $160 \mathrm{~ns}$ after the initiation of the discharge in (a) a mixture of 2 bar He/60 mbar Ar/1.5 mbar $\mathrm{F}_{2}$, (b) same mixture as in (a), but with 0.53 mbar (400 mTorr) Xe added, (c) a mixture of 2 bar Ne/1.5 mbar $F_{2} / 60$ mbar Ar and (d) same mixture as in (c), but with 0.53 mbar (400 mTorr) Xe added. The pump power density and the current density in (a) and (b) are about $1.3 \mathrm{MW} \mathrm{cm}^{-3}$ and $340 \mathrm{~A} \mathrm{~cm}^{-2}$, respectively. In (c) and (d), they are about $410 \mathrm{~kW} \mathrm{~cm}^{-3}$ and $390 \mathrm{~A} \mathrm{~cm}^{-2}$, respectively

proximately $80 \mathrm{~ns}$ weak filaments appeared in the discharge. Up to $160 \mathrm{~ns}$, the filamentation of the discharge increased only slightly, after which, at 200 ns (image not shown in Fig. 3(b)), the homogeneity of the discharge was seriously deteriorated. The addition of more Xe up to partial pressures of $1.53 \mathrm{mbar}$ did not improve the discharge homogeneity further. Figure 3(c) shows images of the discharges in a $\mathrm{Ne}$ buffered ArF excimer laser gas mixture. It can be seen that this discharge was homogeneous up to about $120 \mathrm{~ns}$. Thereafter, filaments appeared in the bulk of the discharge and by $160 \mathrm{~ns}$ the discharge became rather non-uniform. It could be seen also from these discharge pictures that at $80 \mathrm{~ns}$ the discharge current went through a single hot spot at the cathode and was confined to a small area towards the end of the pump pulse. When comparing Fig. 3(a) and 3(c) it can be seen that the discharges in Ne buffered gas mixtures are slightly less filamentary than the discharges in He buffered gas mixtures. In contrast to this, Fig. 3(d) shows that the addition of 0.53 mbar (400 mTorr) Xe to a Ne buffered gas mixture homogenised the discharge substantially and the discharge homogeneity was preserved for about 200 ns. Our experiments proved that a significant extension of the duration of the discharge homogeneity was achieved by adding small amounts of Xe.
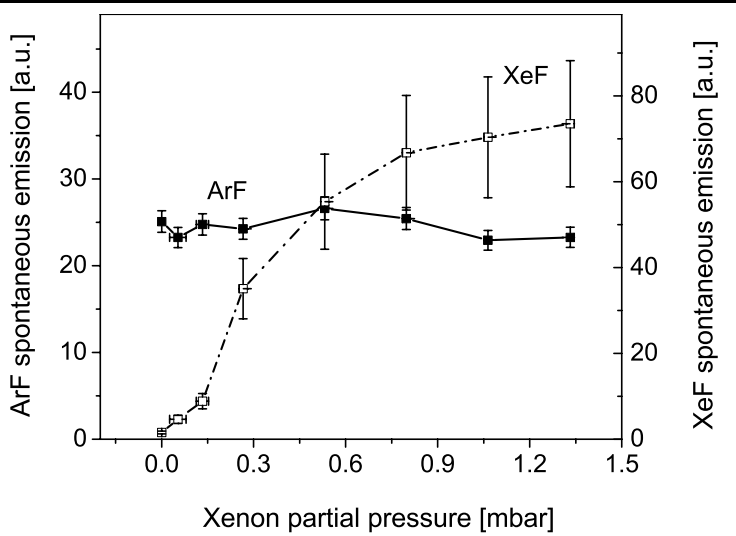

Fig. 4 Spontaneous emission of $\mathrm{ArF}$ and $\mathrm{XeF}$ excimers versus the Xe partial pressure added to the laser gas mixture. The base gas mixture contained 2 bar $\mathrm{He}, 60 \mathrm{mbar} \mathrm{Ar}$ and 1.5 mbar $\mathrm{F}_{2}$. The pump power density and the current density amounted to be $1.3 \mathrm{MW} \mathrm{cm}^{-3}$ and $340 \mathrm{~A} \mathrm{~cm}^{-2}$, respectively

Applying this technique in for example ArF excimer lasers one has to be sure that the addition of Xe does not influence the formation kinetics of ArF excimers in a negative way. The addition of $\mathrm{Xe}$ to the commonly used $\mathrm{ArF}$ or $\mathrm{KrF}$ excimer laser gas mixtures will inevitably lead to the formation of $\mathrm{XeF}$ excimers which may hinder or even compete with the formation of $\mathrm{ArF}$ excimers in $\mathrm{He}$ (or $\mathrm{Ne}$ ) $/ \mathrm{Ar} / \mathrm{F}_{2}$ discharges. Such a new formation channel in the kinetic chain could result in a lower ArF laser gain and efficiency. In order to check this, the spontaneous emission signals from $\mathrm{ArF}$ (at $193 \mathrm{~nm}$ ) and $\mathrm{XeF}$ (at $351 \mathrm{~nm}$ ) excimers have been simultaneously monitored for increasing partial pressures of $\mathrm{Xe}$ in $\mathrm{He} / \mathrm{Ar} / \mathrm{F}_{2}$ gas mixtures. Figure 4 shows the integral of the spontaneous emission signals of $\mathrm{ArF}$ and $\mathrm{XeF}$ (which corresponds to the energy of the spontaneous emission) versus the partial pressure of Xe for the base gas mixtures of 2 bar He, 60 mbar Ar and 1.5 mbar $F_{2}$. It can be seen that the ArF spontaneous emission remained nearly constant while the spontaneous emission from $\mathrm{XeF}$ excimers indeed increased with increasing Xe pressure. One could expect that above some Xe partial pressure the formation of ArF would be suppressed in favor of the $\mathrm{XeF}$ formation. This effect should be seen as a decrease of the ArF spontaneous emission signal, however as can be seen from our measurements, this limit was not reached with the Xe pressures used in our experiments. To investigate if an improvement in discharge homogeneity also can be achieved by the addition of a different heavy rare gas, we also added $\mathrm{Kr}$ to the used laser gas mixtures. We found that the addition of krypton, up to partial pressures of 1.3 mbar did not improve the discharge uniformity in ArF excimer laser gas mixtures.

The important conclusion from our investigations on the discharge homogeneity in fluorine-based excimer laser gas mixtures is that addition of small amounts of Xe to these mixtures is a powerful tool to produce stable long pulse glow 
discharges in $\mathrm{ArF}$ laser gas mixtures and that the formation of $\mathrm{ArF}$ excimer molecules is unaffected by this addition.

\section{Discussion}

In a paper by Gerritsen et al. [11] in 1990, it was shown for the first time that small amounts of Xe added to pure Ne have a very strong impact on the charge density produced by direct photoionization due to X-rays. There was an almost discontinuous increase in charge density for mixtures of neon and xenon, the latter added at very low concentrations as compared with pure neon. In 1995 Taylor et al. [12] studied extensively the photoionization effects by VUV light in rare-gas halide mixtures by adding small amounts of different gases. They found that these additions undoubtedly led to an increase in charge density in the gas mixtures. The relatively large numbers of patents granted shortly after the publication of this paper about adding small amounts of gases with low ionization potentials to excimer laser gas mixtures are probably after effects of this paper. As mentioned before, all the published results are for very short pulse duration systems not exceeding 60-70 ns. We, on the other hand, describe in the present paper very striking discharge improving effects also after $70 \mathrm{~ns}$ from the start of the discharge. The discharge homogeneity in halogen doped raregas mixtures that are usually used in excimer laser systems is improved enormously by adding small amounts of impurities like xenon to the clean laser gas mixtures. The amounts of added Xe needed to show optimal discharge stability enhancements were small enough not to influence the kinetic processes leading to excimer formation. This was experimentally verified by the presented data of the spontaneous emission signals emitted by the different laser gas mixtures. It is assumed that addition of small amounts of Xe to the excimer laser gas mixtures lead to two additional processes that may contribute to a significant enhancement of the charge density and thus to more stable and homogeneous discharge. Due to the low ionizing potential of Xe $(12.1 \mathrm{eV})$ direct photoionization by the X-rays at the beginning of the discharge and by VUV photons created throughout the discharge produce additional charges inside the laser gas mixture. It is well known that the photoionization cross section of Xe is very large for VUV photons. Other possible channels to enhance the charge density in the laser gas mixture are Penning ionization processes with low ionization molecules. Penning ionization is an indirect photo-ionization process. For this process, excited $\mathrm{Xe}$ atoms are required which transfer their energy to the low ionization molecules leading to ionization of these molecules. Excited Xe atoms are created by the discharge itself throughout the whole discharge period. The creation of new charge carriers by these processes and the generation of new electron avalanche channels may explain the very positive effects on the discharge homogeneity later on in the discharge. Both assumptions can be checked either by detecting the spontaneous emitted light spectrum down into the VUV or by studying the role of impurities in the gas mixture more carefully.

\section{Conclusion}

In summary, we presented experimental results showing that the addition of low partial pressures of xenon to $F_{2}$-based excimer laser gas mixtures improved strongly the uniformity of long pulse discharges. In addition, the extra Xe extended the stable phase of the discharge to durations of up to $200 \mathrm{~ns}$. Simultaneous monitoring of the spontaneous emission of $\operatorname{ArF}(193 \mathrm{~nm})$ and $\mathrm{XeF}(351 \mathrm{~nm})$ excimers revealed that the addition of xenon did not perturb the formation of $\mathrm{ArF}$ excimer molecules. Therefore, the addition of $\mathrm{Xe}$ to $\mathrm{F}_{2}$-based excimer laser gas mixtures can be employed for the development of long pulse $\mathrm{F}_{2}, \mathrm{ArF}$ and $\mathrm{KrF}$ lasers with high beam quality.

Acknowledgements This research is supported by the Netherlands Technology Foundation (STW), The Applied Science Division of NWO and the Technology Programme of the Ministry of Economic Affairs.

Open Access This article is distributed under the terms of the Creative Commons Attribution Noncommercial License which permits any noncommercial use, distribution, and reproduction in any medium, provided the original author(s) and source are credited.

\section{References}

1. R.S. Taylor, Preionization and discharge stability study of long optical pulse duration UV preionized XeCl lasers. Appl. Phys. B 41, 1-24 (1986)

2. M.J. Kushner, Microarcs as a termination mechanism of optical pulses in electric-discharge-excited $\mathrm{KrF}$ lasers. IEEE Trans. Plasma Sci. 19, 387-399 (1991)

3. H.M.J. Bastiaens, Ph.D. thesis, ISBN: 90365141 69, University of Twente (2000)

4. L. Feenstra, Ph.D. thesis, ISBN: 90365133 83, University of Twente (1999)

5. N. Kataoka, M. Itagaki, K. Uchino, K. Muraoka, A. Takahashi, T. Okada, M. Maeda, T. Hori, K. Terashima, A. Suitani, T. Enami, H. Mizoguchi, Jpn. J. Appl. Phys. 38, 6735 (1999)

6. H. Besaucele, T. Ishihara, T. Hofmann, Patent, WO 01/08278 A1 (2001)

7. S. Tanaka, K. Kakizaki, Y. Sasaki, US Patent, 2002/0122449 A1 (2001)

8. K. Terashima, A. Sumitani, E. Sunaka, US Patent, 7006548 (2006)

9. D. Mathew, H.M.J. Bastiaens, K.J. Boller, P.J.M. Peters, Appl. Phys. Lett. 88, 101502 (2006)

10. D. Mathew, H.M.J. Bastiaens, K.J. Boller, P.J.M. Peters, J. Appl. Phys. 102, 033305 (2007)

11. J.W. Gerritsen, H.P. Godfried, R.J.M. Bonnie, J. Appl. Phys. 67, 2716 (1990)

12. R.S. Taylor, K.E. Leopold, IEEE J. Quantum. Electron. 31, 2195 (1995) 